\title{
Commentary on "Signal intensity ratio of draining vein on silent MR angiography as an indicator of high-flow arteriovenous shunt in brain arteriovenous malformation"
}

\author{
$\operatorname{Li~Ma} a^{1,2}$ (1)
}

Received: 2 July 2021 / Revised: 1 August 2021 / Accepted: 14 August 2021 / Published online: 15 October 2021

(c) European Society of Radiology 2021

The high-flow arteriovenous (A-V) shunt in arteriovenous malformation (AVM) has been found to be associated with increased hemorrhage risk and incomplete obliteration after radiosurgery [1]. The hemodynamic and angioarchitectural features were also potential target and tailored individually in surgical and endovascular treatment. This preliminary study introduced a non-invasive approach to evaluate the high-flow A-V shunt and revealed a negative correlation between venous signal intensity on silent MRA and transit time of blood flow through AVM nidus on DSA [2]. A draining vein of high signal intensity on silent MRA suggests fast blood flow through AVM nidus. With the evidence of feasibility from this study, a color-coding of the quantitative MRA with appropriate cut-off value would be optimal for outcome assessment after radiosurgery and embolization, or designing multi-modality treatment.

It is interesting to find some novel risk features of AVM rupture focusing on the draining vein or outflow of the lesion [3]. With recent advances of quantitative imaging analysis, accumulating studies unraveled the unbalanced inflow and outflow in AVM with higher hemorrhage risk. The stasis index (a ratio of inflow gradient and outflow gradient) of the dominant draining vein on quantitative DSA was correlated with AVM hemorrhage [4]. Our angiogram data suggested a fast inflow along with restricted venous outflow structure was predictive of future hemorrhage risk. The present study also confirmed a higher ratio of undilated draining vein with high signal intensity or fast flow though AVM lesion in ruptured AVM [2]. The fast flow through AVM

Li Ma

marygl@hotmail.com

1 Department of Neurosurgery, Beijing Tiantan Hospital, Capital Medical University, 119 W Ave 4th S Ring Rd, Fengtai District, 100079 Beijing, People's Republic of China

2 China National Clinical Research Center for Neurological Diseases, Beijing, People's Republic of China nidus was found to be correlated with reduced mural cell coverage of AVM vessels and may contribute to vascular instability in AVM with hemorrhage [5]. This silent MRA study of AVM presented the relationship between venous signal intensity and the transit time through nidus [2], which supplement the spatial resolution of hemodynamic analysis on angiogram. However, it should be noted that the signal intensity and transit time were not derived from the same vascular segment, and the accurate meaning of signal intensity on silent MRA was not fully understood. Further studies with quantitative MRA would be promising to identify more spatio-temporal correlation between signal intensity and hemodynamic features. A fusion of multi-modal imaging techniques to enhance spatio-temporal resolution could be helpful to determine the points with the most significant hemodynamic stress within the AVM lesion, and thereby improve the surveillance of subtle changes predisposing AVM rupture during follow-up.

The region of interest (ROI) is important for interpreting the measurement in quantitative imaging analysis and should be noted when comparing different studies. The feeding arteries and drainage veins were the most common ROIs in understanding AVM hemodynamics. To standardize the quantification, normal vessels (the ipsilateral cavernous segment of the internal carotid artery) would be used as reference. Although the AVM nidus could be more susceptible to vascular injury and the real "region of interest" for this disease, it used to be too tortuous and overlapped to be set as ROI with manual selection. The 3D quantitative MR sequence would provide more possibilities to understand the characteristics and changes within the AVM vasculature [6]. However, with manual placement of ROI, interobserver reliability in deriving parametric imaging measurements could be unsatisfactory that minor differences among raters may result in a large variance [7]. Therefore, further attempt to deconstruct the AVM nidus and to reduce interobserver variance would be of value. Deep learning and 
artificial intelligence might help in automatically learning features and patterns from massive data of serial ROI in AVM vasculature.

Funding The authors state that this work has not received any funding.

\section{Declarations}

Guarantor The scientific guarantor of this publication is Yuanli Zhao at Beijing Tiantan Hospital.

Conflict of interest The authors of this manuscript declare no relationships with any companies whose products or services may be related to the subject matter of the article.

Statistics and biometry No complex statistical methods were necessary for this paper.

\section{Informed consent None.}

Ethical approval Institutional review board approval was not required because this is an editorial comment.

\section{Methodology}

- Comment

\section{References}

1. Taeshineetanakul P, Krings T, Geibprasert S et al (2012) Angioarchitecture determines obliteration rate after radiosurgery in brain arteriovenous malformations. Neurosurgery 71:1071-1078; discussion 1079

2. Wu CX, Zang ZX, Hong T et al (2021) Signal intensity ratio of draining vein on silent MR angiography as an indicator of highflow arteriovenous shunt in brain arteriovenous malformation. Eur Radiol. https://doi.org/10.1007/s00330-021-08170-8

3. Burkhardt JK, Chen X, Winkler EA, Cooke DL, Kim H, Lawton MT (2017) Delayed venous drainage in ruptured arteriovenous malformations based on quantitative color-coded digital subtraction angiography. World Neurosurg 104:619-627

4. Lin TM, Yang HC, Lee CC et al (2019) Stasis index from hemodynamic analysis using quantitative DSA correlates with hemorrhage of supratentorial arteriovenous malformation: a crosssectional study. J Neurosurg 132:1574-1582

5. Ma L, Chen XL, Chen Y, Wu CX, Ma J, Zhao YL (2017) Subsequent haemorrhage in children with untreated brain arteriovenous malformation: higher risk with unbalanced inflow and outflow angioarchitecture. Eur Radiol 27:2868-2876

6. Schneider TM, Mohlenbruch M, Denoix M et al (2020) Susceptibility-based characterization of cerebral arteriovenous malformations. Invest Radiol 55:702-710

7. Narsinh KH, Mueller K, Nelson J et al (2020) Interrater reliability in the measurement of flow characteristics on color-coded quantitative DSA of brain AVMs. AJNR Am J Neuroradiol 41:2303-2310

Publisher's note Springer Nature remains neutral with regard to jurisdictional claims in published maps and institutional affiliations. 